\title{
Art and Science of Instrument Development for Stated-Preference Methods
}

\author{
Ellen M. Janssen $^{1,2} \cdot$ John F. P. Bridges ${ }^{1,2}$
}

Published online: 14 June 2017

(c) Springer International Publishing AG 2017

Ryden et al. [1] contribute to a growing literature on instrument development as it pertains specifically to statedpreference methods. In their article "Discrete choice experiment attribute selection using a multinational interview study: treatment features important to patients with type 2 diabetes mellitus", they describe how they used both literature reviews and qualitative methods to inform the development of a discrete-choice experiment. Such articles have received more attention as researchers seek to be more transparent on the development of their studies [2-4]. Guidance by the US Food and Drug Administration has emphasized the need for patient-preference studies to be patient centered, relevant, and understandable with limited bias in the interpretation of choice tasks [5]. These types of study qualities can only be ensured through deliberate instrument development.

It has been increasingly recognized that instrument development is crucial to ensure that important issues in stated-preference instruments are correctly specified and that choice tasks elicit patient preferences accurately [6]. Despite existing rigorous standards for stated-preference methods [7], including recommendations for experimental design [8] and statistical analysis [9], Ryden et al. rightly note that there is little in the way of consensus-based

This editorial refers to the article available at doi:10.1007/s40271017-0225-0.

Ellen M. Janssen

ejanssen@jhu.edu

1 Department of Health Policy and Management, Johns Hopkins Bloomberg School of Public Health, 624 N. Broadway, Rm. 690, Baltimore, MD 21205, USA

2 Johns Hopkins Center of Excellence in Regulatory Science and Innovation, Baltimore, MD, USA standards for instrument development. Standards on instrument development would emphasize the importance of the instrument development process and enable more clarity and consistency in reporting [6].

In recent years, several research groups have developed and shared frameworks for the development of statedpreference instruments. Coast et al. describe instrument development as the identification and refinement of attributes [10]. Kløjgaard et al. discuss the value of a qualitative process [11]. DosReis et al. discuss rigorous qualitative methods to select, validate, and prioritize attributes [12]. Janssen et al. propose a five-step framework that can be adapted to fit the research objective of different studies [13]. Additionally, Hollin et al. discuss strategies to engage various stakeholders in instrument development [14].

Different instrument development processes emphasize different strategies, have different objectives, and can contain different approaches, as presented in Fig. 1. These different approaches take advantage of the knowledge and experience of various stakeholders, such as patients, caregivers, clinicians, regulators, or industry representatives. Not one instrument development process is necessarily preferred over the other and processes should be selected based on the research objective and question. We have found the greatest success in instrument development for our stated-preference studies by combining different processes and by incorporating the perspectives of different stakeholders [13].

Evidence reviews can optimize the existing knowledge on a topic of interest. Evidence can be extracted from clinical trials if the objective of the research is to compare two competing treatments or medical devices [15]. Attributes and levels can also be directly extracted and synthesized from published preference studies, an approach Ryden et al. took to identify potential attributes for 
Fig. 1 Processes employed in the instrument development of stated-preference studies

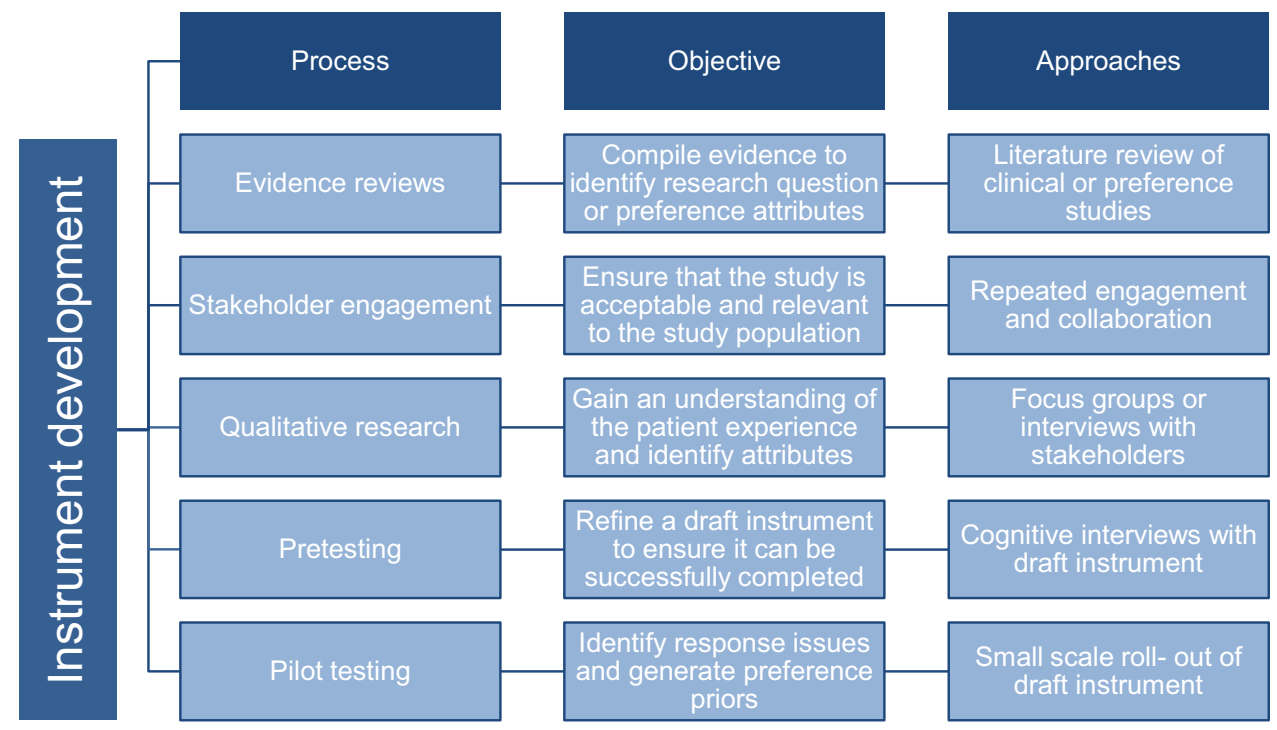

treatment preferences in diabetes mellitus. They did not consider the literature reviews and the synthesis of attributes that had already been conducted on preferences and diabetes [13, 16-18].

Qualitative methods during the development process can serve to gain an increased understanding of the patient experience and to identify a range of attributes that might play into treatment choices. The most common qualitative research methods employed in instrument development are focus groups [3] or one-on-one interviews [19]. Ryden et al. showed that by carefully considering the attribute identification process, including cultural factors, it is possible to conduct extensive qualitative work across different countries. Their study process might have been facilitated through the engagement of stakeholders in each of the study countries before interviews commenced. During engagement, stakeholders are repeatedly engaged with the study throughout all stages of instrument development and have an active voice in research conduct [14]. Stakeholder engagement can be especially useful to ensure that the development process and the instrument itself are acceptable and relevant to the study population of interest [20].

After attribute identification, the next step in the instrument development process concerns attribute refinement [10]. This often includes pretest interviews and pilot testing [13]. Pretesting generally consists of cognitive interviews with research participants focused around refining a draft instrument. Pretesting can help narrow down the set of attributes, can identify issues with the description of attributes to minimize miscomprehension, and can help identify issues that can impede participants from successfully completing the choice tasks [2, 3]. Pilot testing is a small-scale roll-out of the developed survey instrument. It can be used to demonstrate feasibility of a preference study in a new research area and generate hypotheses for relative preference weights [21]. Pilot testing can also reveal problems with the quantitative patterns in responses, identify dominant attributes or attributes that are insensitive to level differences, or generate priors on the preference estimates that can be used for the experimental design of the full-scale survey [13].

More researchers need to follow the example set by Ryden et al. and report on instrument development separately from preference estimation results. Standards on instrument development are needed to ensure that this is done in systematic, valid, and replicable manner. Transparently reporting on and publishing the instrument development process ensures that studies undergo some form of quality control through the peer review process. Presentation of developmental processes through peer-reviewed publications, conferences, and other professional forums can also help disseminate these results [22]. Now that a base literature on instrument development exists, researchers need to be sure to consult this growing literature. This will ensure that they are grounding their developmental work in evolving good research practices and that innovation does not happen in a vacuum. Publishing on the instrument development process in detail is essential in advancing stated-preference methods and ensuring highquality stated-preference studies.

\section{Compliance with Ethical Standards}

Funding This editorial was supported by a Patient-Centered Outcomes Research Institute Methods Award (ME-1303-5946), the Johns Hopkins Center of Excellence in Regulatory Science and Innovation, and the US Food and Drug Administration (UO1FD004977). 
Conflict of interest Ellen M. Janssen and John F. P. Bridges have no conflicts of interest directly relevant to the content of this editorial.

\section{References}

1. Ryden A, Chen S, Flood E, Romero B, Grandy S. Discrete choice experiment attribute selection using a multinational interview study: treatment features important to patients with type 2 diabetes mellitus. Patient. 2017. doi:10.1007/s40271-017-0225-0. [Epub ahead of print].

2. Oakes AH, Garmo V, Bone LR, Longo DR, Segal JB, Bridges JFP. Developing a stated-preference instrument to quantitatively assess the barriers and facilitators to the self-management of type 2 diabetes. Patient. 2017. doi:10.1007/s40271-017-0248-6. [Epub ahead of print].

3. Wittenberg E, Bharel M, Saada A, Santiago E, Bridges JF, Weinreb L. Measuring the preferences of homeless women for cervical cancer screening interventions: development of a bestworst scaling survey. Patient. 2015;8(5):455-67. doi:10.1007/ s40271-014-0110-z.

4. O’Hara NN, Roy L, O’Hara LM, Spiegel JM, Lynd LD, FitzGerald JM, et al. Healthcare worker preferences for active tuberculosis case finding programs in South Africa: a best-worst scaling choice experiment. PLoS One. 2015;10(7):e0133304.

5. Unites States Food and Drug Administration. Patient preference information-voluntary submission, review in premarket approval applications, humanitarian device exemption applications, and de novo requests, and inclusion in decision summaries and device labeling. 2016. http://www.fda.gov/downloads/ medicaldevices/deviceregulationandguidance/ guidancedocuments/ucm446680.pdf. Accessed 8 June 2017.

6. Vass C, Rigby D, Payne K. The role of qualitative research methods in discrete choice experiments: a systematic review and survey of authors. Med Decis Making. 2017;37(3):298-313.

7. Bridges JF, Hauber AB, Marshall D, Lloyd A, Prosser LA, Regier DA, et al. Conjoint analysis applications in health: a checklist. A report of the ISPOR Good Research Practices for Conjoint Analysis Task Force. Value Health. 2011;14(4):403-13. doi:10. 1016/j.jval.2010.11.013

8. Johnson FR, Lancsar E, Marshall D, Kilambi V, Mühlbacher A, Regier DA, et al. Constructing experimental designs for discretechoice experiments: report of the ISPOR Conjoint Analysis Experimental Design Good Research Practices Task Force. Value Health. 2013;16(1):3-13. doi:10.1016/j.jval.2012.08.2223.

9. Hauber AB, Gonzalez JM, Groothuis-Oudshoorn CG, Prior T, Marshall DA, Cunningham C, et al. Statistical methods for the analysis of discrete choice experiments: a report of the ISPOR Conjoint Analysis Good Research Practices Task Force. Value Health. 2016;19(4):300-15. doi:10.1016/j.jval.2016.04.004.

10. Coast J, Al-Janabi H, Sutton EJ, Horrocks SA, Vosper AJ, Swancutt DR, et al. Using qualitative methods for attribute development for discrete choice experiments: issues and recommendations. Health Econ. 2012;21(6):730-41. doi:10.1002/hec. 1739.

11. Kløjgaard ME, Bech M, Søgaard R. Designing a stated choice experiment: the value of a qualitative process. J Choice Model. 2012;5(2):1-18. doi:10.1016/S1755-5345(13)70050-2.

12. DosReis S, Castillo WC, Ross M, Fitz-Randolph M, Vaughn-Lee A, Butler B. Attribute development using continuous stakeholder engagement to prioritize treatment decisions: a framework for patient-centered research. Value Health. 2016;19(6):758-66. doi:10.1016/j.jval.2016.02.013.

13. Janssen EM, Segal JB, Bridges JF. A framework for instrument development of a choice experiment: an application to type 2 diabetes. Patient. 2016;9(5):465-79. doi:10.1007/s40271-0160170-3.

14. Hollin IL, Caroline Y, Hanson C, Bridges JF, Peay H. Developing a patient-centered benefit-risk survey: a community-engaged process. Value Health. 2016;19(6):751-7. doi:10.1016/j.jval. 2016.02.014.

15. Aristides M, Weston AR, FitzGerald P, Le Reun C, Maniadakis N. Patient preference and willingness-to-pay for Humalog Mix 25 relative to Humulin 30/70: a multicountry application of a discrete choice experiment. Value Health. 2004;7(4):442-54.

16. von Arx LB, Kjeer T. The patient perspective of diabetes care: a systematic review of stated preference research. Patient. 2014;7(3):283-300. doi:10.1007/s40271-014-0057-0.

17. Joy SM, Little E, Maruthur NM, Purnell TS, Bridges JF. Patient preferences for the treatment of type 2 diabetes: a scoping review. PharmacoEconomics. 2013;31(10):877-92. doi:10.1007/s40273013-0089-7.

18. Purnell TS, Joy S, Little E, Bridges JF, Maruthur N. Patient preferences for noninsulin diabetes medications: a systematic review. Diabetes Care. 2014;37(7):2055-62. doi:10.2337/dc132527.

19. Bridges JF, Paly VF, Barker E, Kervitsky D. Identifying the benefits and risks of emerging treatments for idiopathic pulmonary fibrosis: a qualitative study. Patient. 2015;8(1):85-92. doi:10.1007/s40271-014-0081-0.

20. Peay HL, Hollin I, Fischer R, Bridges JFP. A community-engaged approach to quantifying caregiver preferences for the benefits and risks of emerging therapies for Duchenne muscular dystrophy. Clin Ther. 2014;36(5):624-37.

21. dosReis S, Ng X, Frosch E, Reeves G, Cunningham C, Bridges JF. Using best-worst scaling to measure caregiver preferences for managing their child's ADHD: a pilot study. Patient. 2014;8(5):423-31. doi:10.1007/s40271-014-0098-4.

22. Wittenberg E. Instrument development in choice experiments. Commentary on: "Applying a framework for instrument development of a choice experiment to measure treatment preferences in type 2 diabetes". Patient. 2016;9(5):379-81. doi:10.1007/ s40271-016-0186-8. 